\title{
Der nächste Schritt gegen nicht-kleinzellige Lungenkarzinome
}

\author{
Bei Patienten mit fortgeschrittenem NSCLC haben Tyrosinkinase-Hemmer (TKI) und Checkpoint- \\ Immuninhibitoren Überlebenszeit und Lebensqualität signifikant gebessert. Die größten Hoff- \\ nungen liegen derzeit auf Kombinationen von modernen Krebs- und Immuntherapeutika. TKI \\ der zweiten Generation, die die Blut-Hirn-Schranke überwinden, sind auch eine Option bei Hirn- \\ metastasen. Die Ganzhirn-Bestrahlung rückt hingegen in den Hintergrund.
}

Nicht-kleinzellige Lungenkarzinome (NSCLC) werden heute in molekulare Subtypen eingeteilt. Zielgerichtet wirkende TKI haben besonders die Prognose von Patienten mit fortgeschrittenen Adenokarzinomen und therapierbaren Genaberrationen gebessert, berichtete Prof. Fred Hirsch von der Universität von Colorado in Aurora. Am häufigsten sind EGFR-Mutationen (1520\%), KRAS-Mutationen (25-30\%) und ALK-Mutationen (510\%). Durch eine First-line-Therapie mit TKI konnte bei Patienten mit EGFR-Mutationen das Gesamtüberleben und das progressionsfreie Überleben im Vergleich zur Chemotherapie signifikant gebessert werden. Resistenzen gegen TKI der ersten Generation wie Gefitinib können durch Substanzen der 2. Generation wie Afatinib und Dacomitinib überwunden werden, so Hirsch. Die größten Hoffnungen für weitere Verbesserungen setzt er in Kombinationstherapien von TKI und AngiogeneseHemmstoffen. Ermutigende Ergebnisse lägen z.B. bereits zur Kombinationstherapie mit Erlotinib und Bevacizumab vor.

\section{Besonders Überwindung Blut-Hirn-Schranke wichtig}

Auch für Patienten mit ALK-Mutationen gibt es inzwischen eine Reihe von spezifisch wirkenden TKI. Sprechen die Patienten auf Substanzen der ersten Generation wie Crizotinib nicht mehr an, können erfolgreich Substanzen der Zweitgeneration wie Ceritinib oder Alectinib (noch nicht zugelassen) eingesetzt werden, sagte Hirsch. Besonders wichtig sei die ZNS-Wirksamkeit dieser Substanzen, die die Blut-Hirn-Schranke überwinden können. Crizotinib hat keinen Einfluss auf die ZNS-Progression.

30\%-40\% der NSCLC-Patienten entwickeln Hirnmetastasen, berichtete Dr. Paula Mulvenna aus London. Sie sind mit den gängigen Methoden nur schwer in den Griff zu bekommen. Eine chirurgische Resektion ist oft nicht möglich und auch die Ganzhirnbestrahlung (WBRT) hat nach den Ergebnissen einer neuen Studie kaum einen therapeutischen Nutzen. In der Multicenterstudie in Großbritannien und Australien wurden jeweils 269 Patienten, die für eine chirurgische Resektion oder stereotaktische Radiotherapie nicht infrage kamen, randomisiert allein mit optimaler Supportivtherapie (inkl. Dexamethason) oder zusätzlich mit WBRT (20 Gray in 5 Tagesfraktionen) behandelt. Weder beim Gesamtüberleben noch der Symptomatik, der Lebensqualität oder dem Steroidbedarf gab es deutliche Unterschiede zwischen beiden Gruppen, berichtete Mulvenna (Mulvenna P et al. Lancet 2016, epub September 4). Im Median überleben die Patienten 9 Wochen. Tendenzielle Vorteile der WBRT zeigten sich nur bei jüngeren Patienten. Die Onkologin hält aufgrund

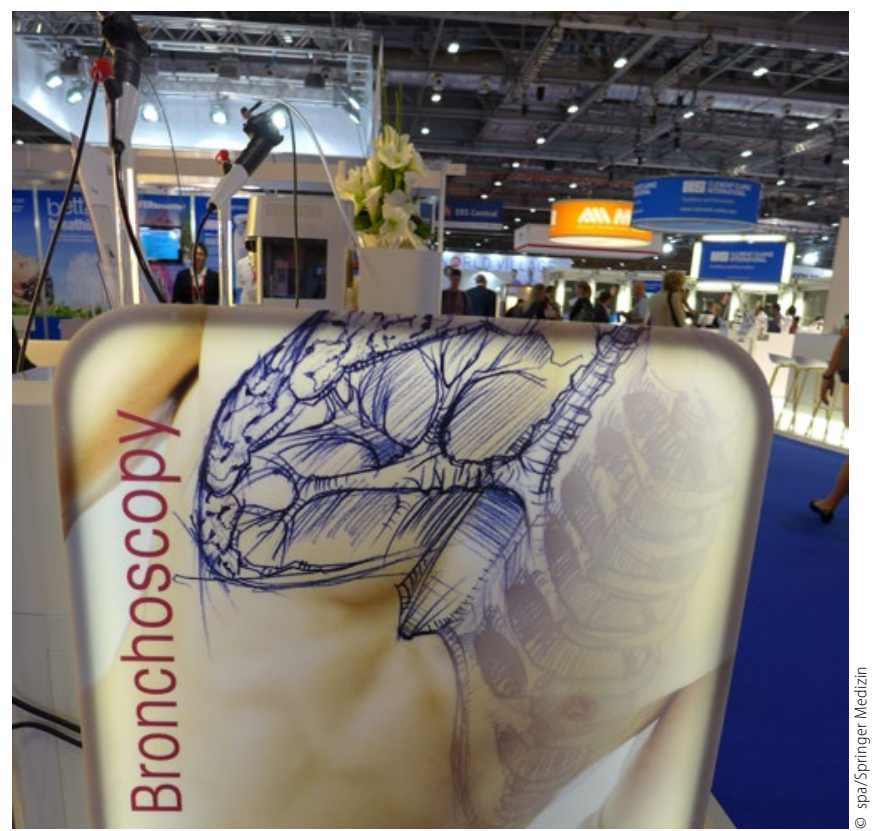

Die Strategie gegen Lungenkrebs hängt maßgeblich von der Charakterisierung des bei der Bronchoskopie biopsierten Tumorgewebes ab.

dieser Daten eine WBRT bei NSCLC-Patienten mit Hirnmetastasen nicht mehr für eine hilfreiche Palliativoption und plädierte für einen Einsatz möglichst nur im Rahmen von Studien.

\section{Wichtiger Fortschritt Checkpoint-Inhibitoren}

Für NSCLC-Patienten ohne therapierbare onkogene Alteration ist die Immuntherapie mit Checkpoint-Inhibitoren, insbesondere über Inhibition des PD-1/PD-L1-Signalwegs (Nivolumab/ Pembrolizumab), ein wichtiger Fortschritt im Vergleich zur Platin-basierten Chemotherapie. Der Signalweg ermöglicht Tumorzellen, sich der Immunabwehr zu entziehen. Am besten sprechen Patienten mit hoher PD-L1-Expression auf PD-1-Hemmer an, so Hirsch, aber auch PD-1/PD-L1-negative Patienten könnten profitieren. Auch bei der Immunonkotherapie gilt die Kombination von PD-1-Hemmern als nächster Schritt zur Verbesserung des Outcomes.

Roland Fath

Quelle: Symposium „Lung cancer: latest developments in screening and patient management", ERS-Kongress 3.-7.9.2016, London 